\title{
TEMPERATURAS DE EXPOSITORES DE ALIMENTOS E QUALIDADE HIGIÊNICO- SANITÁRIA EM RESTAURANTE SELF-SERVICE, NA CIDADE DE ITAPACI-GO
}

\section{TEMPERATURE EXHIBITORS AND SANITARY QUALITY OF FOOD IN SELF-SERVICE RESTAURANT IN THE CITY OF ITAPACI-GO}

\section{Gilmar Aires da Silva}

Docente da FACER Faculdades Unidade de Ceres-GO - Curso de Farmácia

Docente do Instituto Federal de Goiás - campus Uruaçu-GO

Mestre em Química - IQ/UFG - gilmaraires@ hotmail.com

\section{Luciano Alves da Silva}

Docente do Instituto Federal de Goiás - campus Uruaçu-GO

Mestre em Química Analítica - Luciano_tj@yahoo.com

Camilla Chagas Moreira Alves

Faculdade de Farmácia, FACER Faculdades Unidade de Ceres-GO

chagascamila25@hotmail.com

\section{Tatiana Angélica da Costa}

Faculdade de Farmácia, FACER Faculdades Unidade de Ceres-GO

tatinhaangelica@hotmail.com

\section{RESUMO}

Introdução: A modernidade proporciona as pessoas um ritmo acelerado diário fazendo com que os hábitos de vida e alimentares, sejam modificados por vários elementos. Tornando-se, cada vez maior o número de pessoas que se alimentam fora de casa. A comida em restaurante tipo self-service aparece como uma forma de oferecer à população uma refeição mais variada, mais barata e que se assemelha à comida caseira. Por essa razão é imprescindível que se tenha todo um controle do procedimento produtivo que abrange essa modalidade de serviço, obtendo o controle das qualidades de higiene das instalações, dos manipuladores e o controle da temperatura. Sendo assim, este estudo teve como objetivo avaliar temperatura de expositores e qualidade higiênico-sanitárias de alimentos, em dois restaurantes tipo self-service, na cidade de Itapaci - GO. Metodologia: Trata-se de um estudo de campo de caráter qualitativo e quantitativo realizado no período de agosto a setembro de 2015 em dois restaurantes do tipo selfservice em Itapaci-GO. Resultados e discussões: Foram identificados $100 \%$ das coletas, que os alimentos observados nos estabelecimentos pesquisados, estavam em temperaturas inadequadas, apresentaram-se fora da legislação estabelecida a RDC $\mathrm{n}^{\circ}$ 21612004. A média de temperatura para os alimentos quentes foi a recomendação mínima de $60^{\circ} \mathrm{C}$ e as preparações frias inferior a $10^{\circ} \mathrm{C}$. Conclusão: Conclui-se que os restaurantes selfservice analisados possuem falhas na distribuição de seus alimentos, favorecendo o desenvolvimento de microrganismos patogênicos. Portanto, esta irregularidade é considerada crítica na oferta de alimento seguro, com possibilidade de comprometer a qualidade dos alimentos e em seguida ocasionar riscos à saúde do consumidor.

Palavras-chaves: Qualidade higiênico-sanitárias. Manipuladores de alimentos. Checklist. 


\begin{abstract}
Introduction: Modern life gives people an accelerated daily rhythm, causing life and feeding habit being modified by different elements. Becoming, increasingly the number of people who eat away from home. The food in self service style restaurant appears as a way of offering the population a more varied meal, cheaper and which resembles the homemade food. Therefore it is imperative that has an entire control of the production process encompassing this type of service, gaining control of the hygienic quality of the premises, manipulators and the temperature control.Thus, this study aimed to evaluate temperature of the exhibitors and hygiene and sanitary quality of food in two self-service restaurants in the city of Itapaci - GO. Methodology: It is a qualitative field study and quantitative performed over the period August-September 2015 in two self service restaurants in Itapaci-GO. Results and Discusion: Were identified 100\% of the collections, foods observed in the the studied establishments, were in inappropriate temperatures, appearing outside the law established the RDC No. 216 12004 . The average temperature for the warm food was the minimum recommendation of $60{ }^{\circ} \mathrm{C}$ and cold preparations higher $10{ }^{\circ} \mathrm{C}$. Conclusion: The self service restaurants analyzed possess failures in the food distribution, favoring the development of pathogenic microorganisms. Therefore, this irregularity is rated critical in safe food supply with the ability to compromise the quality of foodstuffs and then lead to risks to consumer's health.
\end{abstract}

Keywords: Hygienic and sanitary quality.FoodHandlers. Check-list.

\title{
Endereço para correspondência:
}

Av. Brasil, S/N, Qd. 13, Morada Verde; Ceres-GO

CEP: 76300-000

Fone/Fax: (62) 3323-1040

E-mail: gilmaraires@ hotmail.com

\section{INTRODUÇÃO}

Ao final da década de 1950, no início da emancipação da mulher na sociedade e sua inserção no mercado de trabalho, estimularam o desenvolvimento das cidades e, com isso, foi reduzido o tempo disponível para preparar sua própria refeição. Tornando-se cada vez maior o número de pessoas que se alimentam fora de casa. A comida em restaurante tipo self-service aparece como uma forma de oferecer à população uma refeição mais variada, mais barata e que se assemelha à comida caseira, podendo o consumidor escolher os componentes do seu próprio prato (DE SOUZA GENTA, 2008).

Manter os alimentos para consumo livres de agentes patogênicos depende da capacidade e compromisso de quem os produz e da eficiência das autoridades que legislam e fiscalizam o setor. Assim, a manipulação, armazenagem e exposição destes 
para o consumo devem estar dentro de condições higiênicas e temperaturas adequadas (GONÇALVES, 2013).

A temperatura é um dos fatores que podem influir no crescimento dos microrganismos em alimentos. O processo de cocção e resfriamento de um alimento deve ser realizado de forma a minimizar o risco da multiplicação microbiana e suas consequências ao consumidor final (PAOLONI et al, 2011).

Para garantir a qualidade higiênico-sanitária nos restaurantes, os alimentos, com relação à comercialização, deverão estar em conformidade com que dispõe as Boas Práticas de Fabricação (BPF), que são normas regulamentares estabelecidas pela Agência Nacional de Vigilância Sanitária (ANVISA). Os Procedimentos Operacionais Padronizados (POP) que, de acordo com a Resolução Do Colegiado (RDC) número 275, de 21 de outubro de 2002, são procedimentos que devem estar descritos de forma objetiva e clara, estabelecendo instruções sobre o armazenamento e transporte de alimentos (SIQUEIRA; HAUTRIVE; CEMBRANEL, 2013).

De acordo com a RDC $n^{\circ} 216$, de 15 de setembro de 2004, para não favorecer a multiplicação microbiana, os alimentos deverão ser mantidos em condições de tempo e temperaturas adequadas. Durante a distribuição dos alimentos, as preparações quentes devem ser mantidas a uma temperatura maior que $60^{\circ} \mathrm{C}$, por no máximo 6 horas e as preparações frias devem ser mantidas até $10^{\circ} \mathrm{C}$, por no máximo 4 horas. Quando a temperatura de preparações frias estiver entre $10^{\circ} \mathrm{C}$ e $21^{\circ} \mathrm{C}$, essas preparações só podem permanecer na distribuição por até duas horas. Os alimentos que excedem os critérios de tempo e temperatura determinadosprecisam ser desprezados (GUERREIRO, 2008).

Não estando de acordo com as BPF e a RDC n²1612004, poderão surgir doenças de origem alimentar, que são todas causadas pela ingestão de alimentos que podem estar contaminados com microrganismos patogênicos, substâncias químicas ou que contenham em sua constituição estrutura naturalmente tóxica. Os sintomas mais comuns nesses casos são: diarréia, vômito, dores abdominais, febre, náuseas, sudorese e cefaléia (VERGARA; ALBUQUERQUE, 2011).

Em um restaurante tipo self-service deve-se manter um padrão de temperatura ideal nas bancadas e um controle sobre as condições higiênico-sanitárias, para que os alimentos fiquem em condições ideais para o consumidor. De acordo com a RDC $\mathrm{n}^{\circ}$ 21612004, para não favorecer a multiplicação microbiana, os alimentos deverão ser 
mantidos em condições de tempo e temperatura adequadas. As preparações quentes devem ser mantidas em temperatura superior a $60^{\circ} \mathrm{C}$ por no máximo seis horas e preparações frias, como saladas, devem ser mantidas sob temperatura de refrigeração de até $10^{\circ} \mathrm{C}(\mathrm{CALADO}$ et al, 2009).

Portanto, o foco deste trabalho foi verificar se os quesitos temperatura dos expositores de alimentos e as condições higiênico sanitárias de restaurantes do tipo selfservice do município de Itapaci-GO, estão de acordo com a legislação do setor.

\section{METODOLOGIA}

Trata-se de um estudo de campo de caráter qualitativo e quantitativo realizado no período de agosto a setembro de 2015 em dois restaurantes do tipo self-service em ItapaciGO. Diariamente são servidas as seguintes preparações: saladas, carnes, arroz, feijão e mais uma guarnição. Cada restaurante foi identificado, respectivamente, como A e B. As avaliações das condições higiênico-sanitárias foram de caráter observacional através de um check-list elaborado segundo a RDC 275/2002 - ANVISA - (anexo A).

A pesquisa foi aplicada aos restaurantes mediante a assinatura do termo de consentimento livre e esclarecido pelo proprietário ou gerente de cada restaurante. $\mathrm{O}$ critério de escolha dos restaurantes foi pela colaboração dos donos em participar da pesquisa. Sendo que na cidade de Itapaci-GO há três restaurantes, e só dois concordaram em participar da pesquisa.

As aferições de temperatura foram realizadas com termômetro infravermelho tipo pistola, modelo TD 973 da Termopar Digital com escala de -50 a $+530^{\circ}$ C.Estas foram realizadas o mais perto possível do centro geométrico dos alimentos, em dois dias não consecutivos da semana durante o horário em que as preparações eram servidas. As primeiras aferições foram realizadas antes da abertura dos restaurantes, e as demais durante o transito dos clientes e após a reposição de alimentos nos expositores.

\section{RESULTADOS E DISCUSSÕES}


As temperaturas obtidas estão expressas nas tabelas 1 e 2. Cabe ressaltar que os resultados expressam a média das temperaturas obtidas com uma margem de erro fornecida pelo equipamento de leitura de $\pm 2^{\circ} \mathrm{C}$.

TABELA 1: Temperaturas médias dos expositores do restaurante A antes e depois da abertura.

\begin{tabular}{ccc}
\hline ALIMENTO & ANTES $\left({ }^{\circ} \mathrm{C}\right)$ & DEPOIS $\left({ }^{\circ} \mathrm{C}\right)$ \\
\hline Arroz & $52 \pm 2$ & $45,1 \pm 2$ \\
Feijão & $50,2 \pm 2$ & $52,2 \pm 2$ \\
Carne cozida & $46,5 \pm 2$ & $41,8 \pm 2$ \\
Carne assada & $62,1 \pm 2$ & $49 \pm 2$ \\
Frituras & $52,6 \pm 2$ & $38,2 \pm 2$ \\
Frutas & $21,5 \pm 2$ & $25,1 \pm 2$ \\
Massas & $40,1 \pm 2$ & $38,4 \pm 2$ \\
Legumes cozidos & $19,3 \pm 2$ & $17,5 \pm 2$ \\
Legumes frescos & $15,3 \pm 2$ & $16,1 \pm 2$ \\
\hline
\end{tabular}

TABELA 2: Temperaturas médias dos expositores do restaurante A antes e depois da abertura.

\begin{tabular}{ccc}
\hline ALIMENTO & ANTES $\left({ }^{\circ} \mathrm{C}\right)$ & DEPOIS $\left({ }^{\circ} \mathrm{C}\right)$ \\
\hline Arroz & $51 \pm 2$ & $44,8 \pm 2$ \\
Feijão & $40,4 \pm 2$ & $44,3 \pm 2$ \\
Carne cozida & $47,3 \pm 2$ & $45,1 \pm 2$ \\
Carne assada & $61,1 \pm 2$ & $49,8 \pm 2$ \\
Frituras & $51,1 \pm 2$ & $43,5 \pm 2$ \\
Frutas & $19,4 \pm 2$ & $21,2 \pm 2$ \\
Massas & $37,9 \pm 2$ & $35,8 \pm 2$ \\
Legumes cozidos & $18,6 \pm 2$ & $17,3 \pm 2$ \\
Legumes frescos & $17,6 \pm 2$ & $16,3 \pm 2$ \\
\hline
\end{tabular}


Os resultados desse estudo mostram que as temperaturas médias dos alimentos servidos nos restaurantes self-service da cidade de Itapaci-GO, no momento da coleta estavam inadequadas segundo os parâmetros da RDC - ANVISA - n²1612004, em que a temperatura mínima para preparações quentes deve ser $60^{\circ}$ e para frio até $10^{\circ} \mathrm{C}$.

As unidades avaliadas não possuem o expositor adequado para alimentos resfriados, contribuindo para as temperaturas observadas. Temperaturas fora do padrão estipulado favorecem o desenvolvimento de microrganismos patogênicos, que poderá surgir doenças de origem alimentar, colocando a em risco a saúde do consumidor.

Por conseguinte, um estudo realizado por Silva e Cardoso (2008) em uma unidade de alimentação e nutrição na cidade de Montes Claros-MG, verificou que as temperaturas no balcão de distribuição dos pratos quentes proporcionaram um elevado percentual de amostras fora do padrão instituído pela legislação. A maior porcentagem de não conformidades dentre os pratos quentes ocorreu com o prato principal como arroz, feijão, carne e saladas.

De acordo com Ricardo (2012) todo alimento é susceptível à contaminação por microrganismos desde seu armazenamento até o momento da distribuição. Na distribuição, observou-se que em relação ao arroz e o feijão as temperaturas avaliadas de todos os dois restaurantes pesquisados estavam inadequadas.

Destaca-se que a carne cozida ou assada do mesmo modo necessita manter a temperatura mínima de $60^{\circ} \mathrm{C}$. Contudo, em ambos os estabelecimentos foram encontrados valores inferiores a este. Apesar da unidade B, na primeira avaliação a temperatura da carne estava dentro dos limites, esta não foi mantida durante a exposição, favorecendo a proliferação microbiana. Fidélis (2005) avaliou 97 amostras em estudo no Espírito Santo em cinco restaurantes institucionais, encontrou $15,5 \%$ dos alimentos à base de carne inapropriados para o consumo humano por oferecerem uma ou mais contagens de microrganismos não atendendo à legislação vigorante.

As preparações quentes do tipo frituras também apresentaram variações significativas nas temperaturas em ambas às unidades, podendo indicar temperatura inadequada durante a cocção ou conservação das mesmas.

As frutas devem ficar em lugares com refrigeração, com tempo e temperatura adequados. Se ficarem expostas por muito tempo em condições inadequadas, podem comprometer suas propriedades físico-químicas e nutricionais. Marinho et al, 2009, em 
pesquisa semelhante em Belo Horizonte, observou que as frutas e os legumes ficam expostos sem refrigeração, ainda foi observado que nenhuma das unidades possuía sistema de refrigeração nas bancadas de exposição, favorecendo o escurecimento de frutas, legume e verduras cruas.

Deschamps et al (2003), ressaltam que o controle do binômio tempoltemperatura é de essencial importância para uma maior segurança alimentar no decorrer doprocedimento produtivo, impedindo, deste modo, a proliferação de microrganismos prejudiciais à saúde. De acordo com Deus et al (2005), a temperatura é um dos elementos que mais compromete a viabilidade e a multiplicação microbiana, entretanto o reaquecimento passa a ser a última linha de defesa na prevenção de DTA.

\section{RESTAURANTE A}

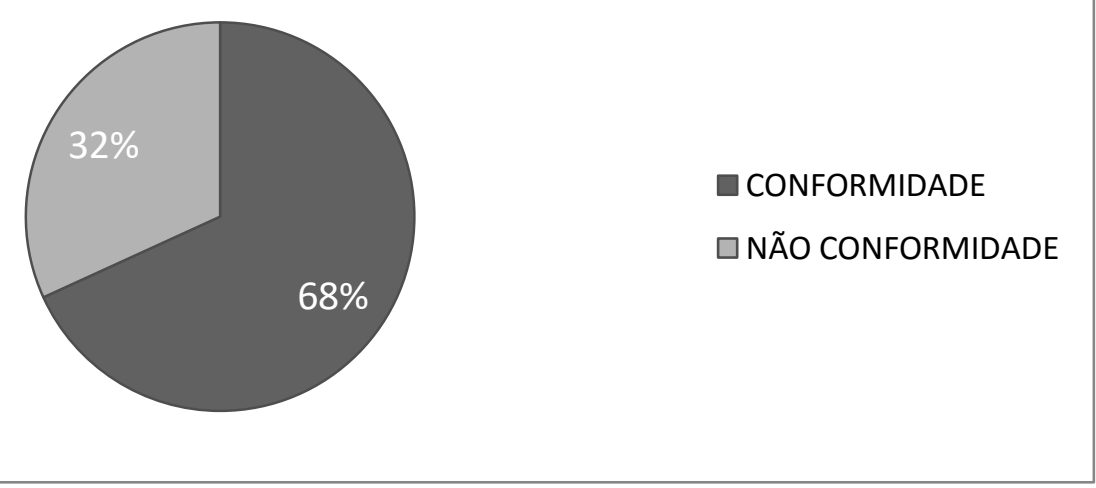

Figura 1-Check-list das condições higiênico-sanitárias aplicado na unidade A.

\section{RESTAURANTE B}

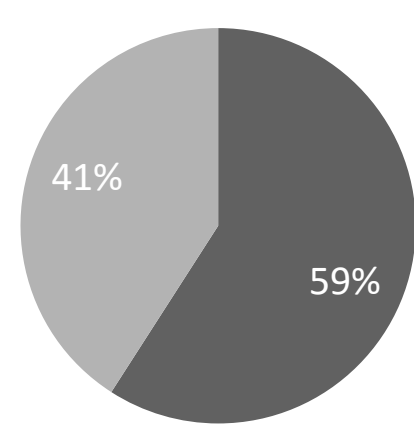

CONFORMIDADE

NÃO CONFORMIDADE

Figura 2- Check-list das condições higiênico-sanitárias aplicado na unidade B. 
De acordo com as tabelas vê-se que no Restaurante " $\mathrm{A}$ " $68 \%$ está em conformidade e $32 \%$ não conformidade. Contudo, no Restaurante "B" 59\% está em conformidade e $41 \%$ não conformidade.

Referente à aparência dos manipuladores descrita pela Vigilância Sanitária (ANVISA), deve-se usar uniforme limpos; não usar adornos; os sapatos devem ser fechados; os cabelos estarem completamente presos e cobertos e as unhas devem estar sempre cortadas, limpas e livres de esmalte. Foi verificado que tanto o restaurante A e B se encontram em conformidade. Esperança et al (2011) menciona que os manipuladores de alimentos são a principal condução de contágio por microrganismos, que habitam e se ampliam em determinadas partes do organismo, principalmente cabelo, nariz, boca, garganta, intestinos, pele e unhas. Assim, é indispensável que os mesmos possuam total higiene no ambiente de trabalho, em especial no local de manipulação dos alimentos.

Ambas as unidades avaliadas estão em conformidade quanto às práticas higiênicas dos manipuladores como não tossir, não espirrar sobre os alimentos, equipamentos e instalações, não levar a mão a qualquer parte do corpo, não cuspir no ambiente, evitando contaminação. Segundo, Pittelkow e Bitello (2014) relatam que os manipuladores devem sempre ter atitudes higiênicas apropriadas no local de trabalho. Assim, a prática da higiene e os comportamentos admitidos no decorrer da manipulação dos alimentos precisam ser comumente supervisionados e desempenhados em capacitações para manipuladores de alimentos. Do mesmo modo, Ramos e Espindola (2006) evidenciam que deve-se sempre evitar conversar, cantar, tossir ou espirrar sobre os alimentos, para que não caia saliva sobre os mesmos. Ribeiro e Schmidt (2007) complementam que a lavagem das mãos e antebraços deve ser frequente, pois a contaminação cruzada pode ocorrer através dos próprios manipuladores de alimentos.

Ao avaliar o local onde armazenam alimentos, verificou que há presença de alimentos encostados na parede, no chão e dentro de caixas de papelão nas duas unidades. Configurando uma não conformidade.

Outra não conformidade encontrada foi a não capacitação dos colaboradores a qual é essencial, tornando-se uma questão de sobrevivência para qualquer empresa. Uma vez que os profissionais devem ser capacitados e prontos para desempenhar sua função de maneira higiênica e segura. Percebeu-se a não conformidade também com a falta de procedimentos operacionais padrão nas unidades, os quais devem ser desenvolvidos para ajudar o 
profissional a assegurar a qualidade de alimentos independente de quem os faça. Segundo Carvalho e Nascimento (2008) a capacitação profissional é essencial ao atual cenário contemporâneo, principalmente entre as empresas que dão preferência aos profissionais capacitados e prontos para desempenhar uma função com eficácia dentro do ambiente empresarial.

Foi encontrado em uma unidade um colaborador usando curativo na mão e tendo contato direto com o alimento. Portanto, esses colaboradores deveriam estar usando luvas para evitar que entre em contato direto com os alimentos ou então serem afastados da manipulação até a sua recuperação, pois, assim, estaria sendo evitada a contaminação cruzada que tem a capacidade de acontecer por meio dos próprios manipuladores de alimentos

O sistema de exaustão é ineficiente para o fluxo de produção, pois esse sistema deve ser de acordo com as necessidades de cada ambiente comercial, devem-seretirar os vapores e gases originados dos procedimentos de cozimento e frituras dos alimentos, fazendo com que o ambiente da cozinha fique livre de odores e fumaça, do mesmo modo como garantir a temperatura interna em condições desejáveis de conforto. De acordo com Paula (2011) todos os estabelecimentos comerciais que trabalham com manipulação de alimentos em cozinhas precisam ter um bom sistema de exaustão, pois diversos problemas podem passar a existir quando uma cozinha não dispersa os odores e a fumaça originada. Além do mais, pode ocorrer contaminações cruzadas e tornar o ambiente insalubre para os colaboradores.

\section{CONCLUSÃO}

Nos resultados indicam que houve falha na manutenção das temperaturas, tanto para o restaurante A, quanto para o restaurante B. Foi verificado que essas duas empresas não seguem o que delimita a RDC n²1612004 e a RDC n²75\2002 ambas ANVISA. Faz-se necessário a melhoria dos equipamentos para a manutenção adequada das temperaturas e ainda melhorar a forma de armazenamento das matérias primas, colocando estas sobre paletes e afastando-as das paredes. Outro ponto está relacionado à necessidade de treinamento dos manipuladores, fato que contribuiria para uma alimentação servida em melhores condições higiênico-sanitárias. Lembrando que a qualidade alimentar está 
relacionada à saúde pública, portanto deve ser imprescindível em qualquer estabelecimento manipulador de alimentos.

\section{REFERÊNCIAS}

BRASIL. Ministério da Saúde. Agência Nacional de Vigilância Sanitária- ANVISA. Cartilha sobre Boas Práticas para serviços de alimentação Resolução-RDC nº 216/2004. Brasília. 2004.

BRUGAlli A; PINTO J. M; TONDO E. C. Análise de perigos e pontos críticos de controle para garantir a segurança alimentar em restaurante da Universidade Federal do Rio Grande do Sul. Revista Higiene Alimentar; v. 16, n. 101, p. 15-19, 2002.

CALADO, I. L; RIBEIRO, M. C. S; FROTA, M. T. B. A; NASCIMENTO, A. R. Avaliação da temperatura dos alimentos na etapa de distribuição em restaurantes selfservice de São Luís, MA. Revista Higiene alimentar,v. 23, n. 174, p. 117-122, 2009.

CARVALHO, Antonio Vieira; NASCIMENTO, Luiz Paulo. Administração de Recursos Humanos. São Paulo: Pioneira, 2008.

DE SOUZA GENTA, T.M; MAURICIO, A.A.; MATIOLI, G. Avaliação das boas práticas através de check-list aplicado em restaurantes self-service da região central de Maringá, estado do Paraná. Acta Scientiarum, v.27, n.2, p. 151-156, 2008.

DESCHAMPS C; FREYGANG J; BRAMORSKI A; TOMMASI D; GARCIA G. F; Avaliação higiênico-sanitária de cozinhas industriais instaladas no município de Blumenal-SC. Revista Higiene Alimentar,v. 17, n. 112, p. 12-15, 2003.

DEUS M. B; HOLLAND N; MORAES C. M. M; STAMFORD T. L. M. Microrganismos patogênicos e temperaturas de exposição de carne bovina servida em restaurantes selfservice da cidade de Natal (RN), Brasil. Revisa,v. 1, n. 4, p. 237-245, 2005. 
ESPERANÇA, M.A. Manual de apoio estabelecimentos de restauração ou de bebidas. Ministério da Saúde, Lisboa, 2011.

FIDÉLIS, G.A. Avaliação das boas práticas de preparação em restaurantes institucionais. Dissertação de Mestrado, 148 f, Viçosa, 2005.

GÓES, J.A.W. et al. Capacitação dos manipuladores de alimentos e a qualidade da alimentação servida Revista Higiene Alimentar, v. 15, n. 82, p. 20-22, 2001.

GONÇALVES, R.M.Frequência de parasitos em alfaces (lactuca sativa) consumidas em restaurantes self-service de Porto Alegre, Rio Grande do Sul, Brasil. Revista Patol. Trop., v. 42, n. 3, p. 323-330, 2013.

GUERREIRO, L. Dossiê técnico. Boas práticas de fabricação em serviços de alimentação. Redetec - Rede de tecnologia do Rio de Janeiro, 2006.

MARINHO C. B; SOUZA C. S; RAMOS S. A. Avaliação do binômio tempo-temperatura de refeições transportadas. e-Scientia,v. 2, n. 1, p. 1-11, 2009.

PAOLONI,D.F; MACEDO, D.C; ALVES, I.C.V. Controle da temperatura de refeições transportadas, em unidades de alimentação e nutrição. Revista Higiene Alimentar, v.25, n. 192, p. 56-60, 2011.

PITTELKOW, A.BITELLO, A.R. A higienização de manipuladores de uma unidade de alimentação e nutrição (UAN). Revista Destaques Acadêmicos, v. 6, n. 3, 2014.

PAULA, C.D.M. Riscos ocupacionais e condições de trabalho em cozinhas industriais. Monografia, 77 f, Porto Alegre, 2011.

RAMOS,A. M; SPINDOLA, R. O. Manual Para Manipuladores de Alimentos. Cartilha 1, Fundação Municipal de Saúde, Teresina, 2006. 
RIBEIRO, K. L.; SCHMIDT, V. Caracterização de manipuladores de alimentos em escolas municipais de Viamão, RS. Revista Higiene Alimentar, São Paulo, v. 21, n. 157, p. 58-64, 2007.

RICARDO, F.O. Controle de tempo e temperatura na produção de refeições de restaurantes comerciais na cidade de Goiânia-GO. Demetra: nutrição \& saúde, v. 7, n. 2, p. 85-96, 2012.

SILVA, B. A. F.; CARDOSO, R. R. Temperatura de alimentos em uma Unidade de Alimentação e Nutrição do município de Montes Claros - MG. Semana da biologia, 2008 .

SIQUEIRA, F; HAUTRIVE, T.P; CEMBRANEL, F. M. Implementação do procedimento operacional padronizado de higienização em restaurante popular de Santa Catarina. Revista Higiene Alimentar; v. 27, n. 216, p. 63-67, 2013.

SOUZA, L.H.L. A manipulação inadequada dos alimentos: fator de contaminação. Revista Higiene Alimentar, v. 20, n. 146, p. 32-39, 2004.

VERGARA, C. M. A. C; ALBUQUERQUE, M. B. Condições higiênico-sanitárias de restaurantes comerciais da cidade de Fortaleza - CE. Revista Higiene Alimentar, v. 25, n. 192, p. 29-34, 2011. 
Anexo A

\begin{tabular}{|c|c|c|}
\hline CHECK LIST & $\mathbf{C}$ & NC \\
\hline $\begin{array}{l}\text { A aparência dos manipuladores de alimentos está de } \\
\text { acordo com as normas descritas pela Vigilância Sanitária } \\
\text { (ANVISA)?(Usa uniforme limpos, não usam adornos, os } \\
\text { sapatos são fechados e o cabelo está completamente preso } \\
\text { e coberto e as unhas encontram-se cortadas, limpas e } \\
\text { livres de esmalte). }\end{array}$ & & \\
\hline $\begin{array}{l}\text { Os manipuladores sempre praticam atitudes higiênicas, } \\
\text { como não tossir, não espirrar sobre os alimentos, } \\
\text { equipamentos e instalações, não levar a mão a qualquer } \\
\text { parte do corpo, não cuspir no ambiente, etc., evitando } \\
\text { contaminação? }\end{array}$ & & \\
\hline $\begin{array}{l}\text { Os manipuladores cumprem as recomendações de lavar e } \\
\text { sanificar as mãos e antebraços sempre que usam o } \\
\text { banheiro? }\end{array}$ & & \\
\hline $\begin{array}{l}\text { Os manipuladores participaram de algum tipo de } \\
\text { treinamento especifico? }\end{array}$ & & \\
\hline $\begin{array}{l}\text { Os manipuladores com curativos nas mãos e braços são } \\
\text { deslocados para serviços que não entrem em contato direto } \\
\text { com os alimentos? }\end{array}$ & & \\
\hline $\begin{array}{l}\text { Os manipuladores obedecem às recomendações de fumar } \\
\text { somente nas áreas destinadas a este fim? }\end{array}$ & & \\
\hline $\begin{array}{l}\text { Os manipuladores não usam perfume que possa transmitir } \\
\text { odor aos alimentos? }\end{array}$ & & \\
\hline $\begin{array}{l}\text { Quanto aaparência do local em que se manipula os } \\
\text { alimentos (cozinha), as portas estão limpas, as janelas } \\
\text { também estão livres de sujidades, o chão não está sujo, as } \\
\text { bancadas que servem de apoio para o preparo dos } \\
\text { alimentos estão adequadamente limpas e sanificadas, o } \\
\text { fogão é devidamente limpo e as lixeiras são de pedais para } \\
\text { manter a higiene adequada do local? }\end{array}$ & & \\
\hline $\begin{array}{l}\text { As instalações estão devidamente projetadas de forma a } \\
\text { facilitar o recolhimento das águas residuais? }\end{array}$ & & \\
\hline
\end{tabular}




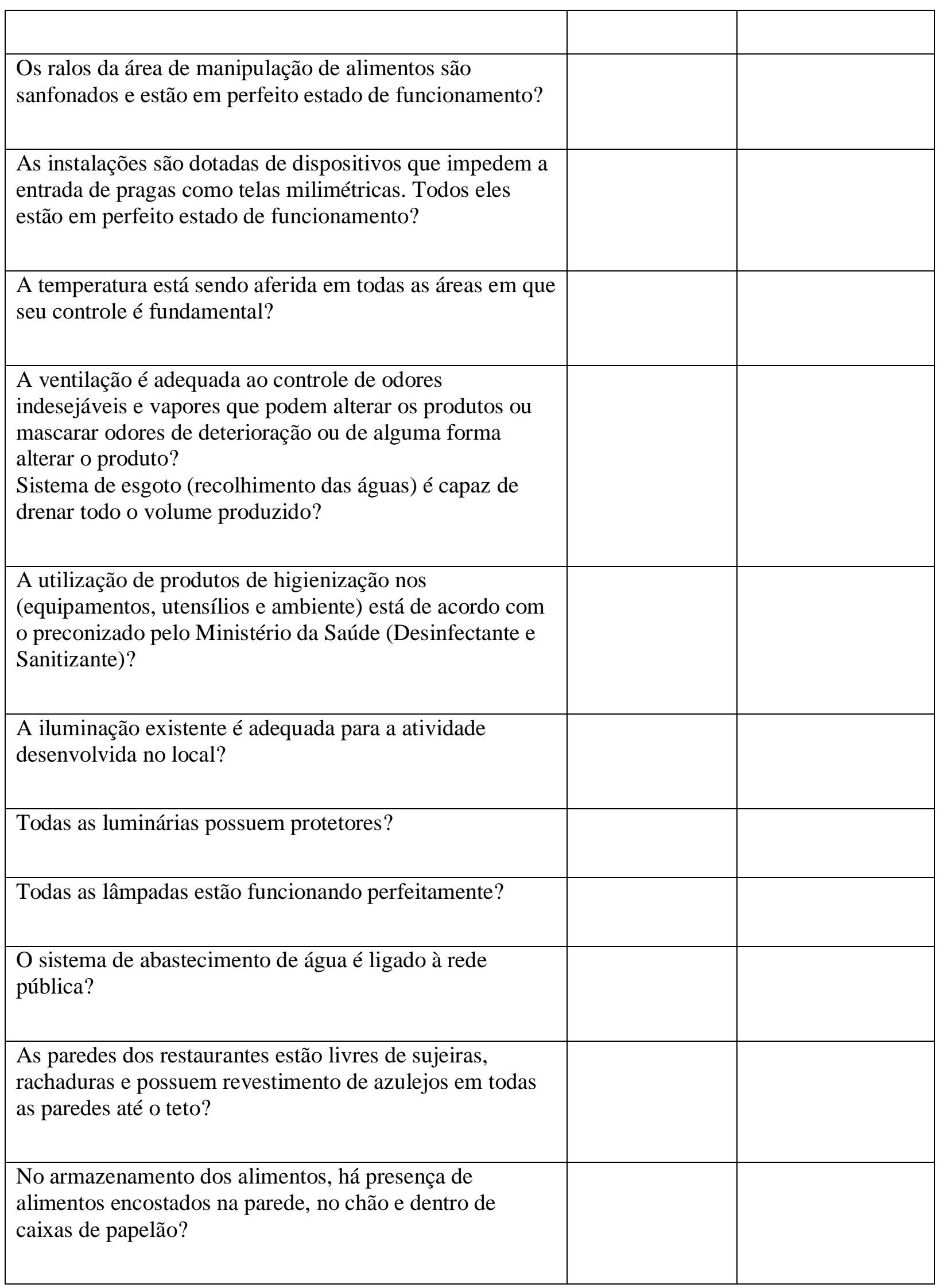


Os alimentos estão separados dos matérias de limpeza e sanificantes?

As geladeiras e os frezzers utilizados para o armazenamento de frios estão em bom estado de conservação e limpos?

Há registros contínuos dos controles de temperatura (Geladeiras e Freezer).

As instalações sanitárias estão dotadas de produtos destinados à higiene pessoal: papel higiênico, sabonete líquido inodoro antisséptico, toalhas de papel não recicladas para as mãos ou outro sistema higiênico e seguro para secagem? 